Louisiana State University

LSU Digital Commons

$10-14-2014$

\title{
Compressive direct measurement of the transverse photonic wavefunction
}

Mohammad Mirhosseini

University of Rochester Institute of Optics

Omar S. Magaña-Loaiza

University of Rochester Institute of Optics

S. M. Hashemi Rafsanjani

University of Rochester

Robert W. Boyd

University of Rochester Institute of Optics

Follow this and additional works at: https://digitalcommons.Isu.edu/physics_astronomy_pubs

\section{Recommended Citation}

Mirhosseini, M., Magaña-Loaiza, O., Hashemi Rafsanjani, S., \& Boyd, R. (2014). Compressive direct measurement of the transverse photonic wavefunction. Frontiers in Optics, FiO $2014 \mathrm{https}$ ://doi.org/ 10.1364/fio.2014.fm4e.5

This Conference Proceeding is brought to you for free and open access by the Department of Physics \& Astronomy at LSU Digital Commons. It has been accepted for inclusion in Faculty Publications by an authorized administrator of LSU Digital Commons. For more information, please contact ir@lsu.edu. 


\title{
Compressive Direct Measurement of the Transverse Photonic Wavefunction
}

\author{
Mohammad Mirhosseini ${ }^{1, *}$, Omar S. Magaña-Loaiza ${ }^{1}$, S. M. Hashemi Rafsanjani ${ }^{2}$, and \\ Robert W. Boyd ${ }^{1,3}$ \\ ${ }^{1}$ The Institute of Optics, University of Rochester, Rochester, New York 14627, USA \\ ${ }^{2}$ Center for Coherence and Quantum Optics and the Department of Physics \& Astronomy, University of Rochester, \\ Rochester, New York 14627, USA \\ ${ }^{3}$ Department of Physics, University of Ottawa, Ottawa ON K1N 6N5, Canada \\ *mirhosse@optics.rochester.edu
}

\begin{abstract}
We generalize the method of direct measurement and combine it with compressive sensing. Using our method, we measure a 19200-dimensional state using only $20 \%$ of the total required measurements.
\end{abstract}

(C) 2014 Optical Society of America

OCIS codes: 270.0270, 270.5585, 100.5070.

Determining an unknown wavefunction is of fundamental importance in quantum mechanics. Despite many seminal contributions, this task remains challenging for high-dimensional states. The direct measurement (DM) approach, introduced by Lundeen et. al, has provided a ground for meeting the high-dimensionality challenge [1]. Contrary to state tomography, this methods does not require a time-consuming post-processing. Nevertheless, the number of measurements required by the direct measurement protocol grows linearly with the dimensionality of the measured state. Here we combine a novel computational method known as compressive sensing with the direct measurement technique. Utilizing our approach, the wavefunction of a high-dimensional state can be estimated with a high fidelity using much fewer number of measurements compared to the standard direct measurement.
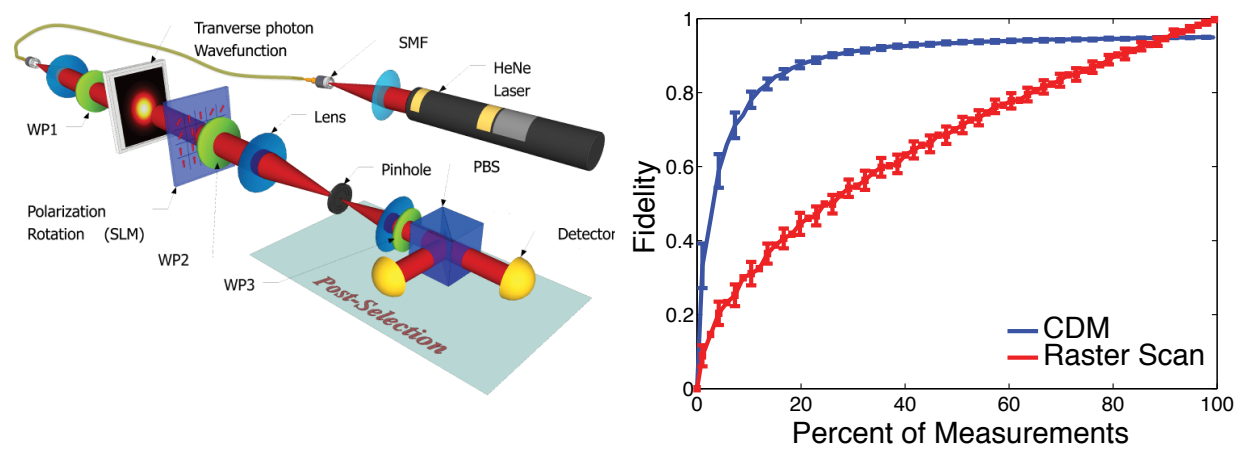

Fig. 1. A schematic illustration of the experimental setup (left). The fidelity of a reconstructed Gaussian state with the target wavefunction, shown in blue, as a function of the percentage of the total measurements (right). The fidelity of the state reconstructed from a partial pixel-by-pixel scan with the same number of measurements is shown in red for comparison.

A weak value is the expectation value of a weak measurement that is followed by a post-selection [2]. Consider a weak measurement of the position projector $\hat{\pi}_{j}=\left|x_{j}\right\rangle\left\langle x_{j}\right|$ at point $x_{j}$ followed by a post-selection on the zeroth component of the Fourier transform of the spatial wavefunction, which we denote by $|o\rangle$. The complex wavefunction of a photon can be calculated at each point by measuring the real and imaginary part of the weak value as

$$
\pi_{w}=\frac{\left\langle o \mid x_{j}\right\rangle\left\langle x_{j} \mid \psi\right\rangle}{\langle o \mid \psi\rangle}=\frac{\psi\left(x_{j}\right)}{\phi_{0} \sqrt{N}} .
$$



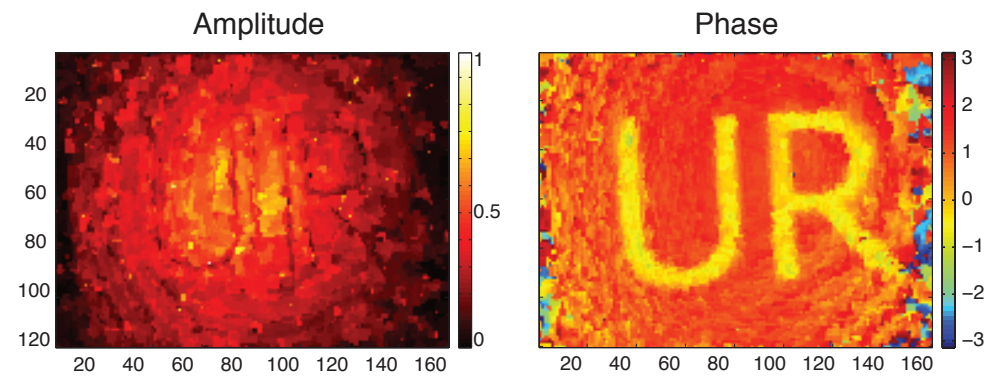

Fig. 2. The amplitude and phase of a Gaussian mode illuminating a custom phase mask (the initials of the University of Rochester). The data is reconstructed by the CDM method with $\mathrm{N}=19200$, and $M / N=20 \%$ of total measurements.

Here we have used the Fourier transform property $\left\langle o \mid x_{j}\right\rangle=1 / \sqrt{N}$ where $N$ is the dimension of the Hilbert space and $\phi_{0}=\langle o \mid \psi\rangle$.

We generalize the DM to a form suitable for compressive sensing. Let the initial system-pointer state be $|\Omega\rangle=$ $|\psi\rangle \otimes|V\rangle=\sum_{i=1}^{N} \psi_{i}\left|x_{i}\right\rangle \otimes|V\rangle$, where we have assumed to have a discrete Hilbert space for the spatial degree of freedom $|\psi\rangle$ and a two-level system such as the polarization of a single photon for the pointer state $|V\rangle$. We consider a situation where instead of a measuring a projector $\hat{\pi}_{j}$ we perform a weak measurement of the operator $\hat{Q}_{m}=\sum_{j} Q_{m, j} \hat{\pi}_{j}$ where the coefficients $Q_{m, j} \in \mathbb{R}$. In this situation the imaginary and the real part of $\psi_{j}, \mathfrak{I}\left[\psi_{j}\right]$ and $\mathfrak{R}\left[\psi_{j}\right]$, can be related to the expectation values of the polarization of the post-selected state $\bar{\sigma}_{x, m}$ and $\bar{\sigma}_{y, m}$ via a linear set of equations $\phi=\mathbf{Q} \psi$.

Here, $\phi_{m}=\frac{1}{\kappa}\left[\bar{\sigma}_{x, m}+i \bar{\sigma}_{y, m}\right]$ and $\kappa=\frac{2 \alpha}{\phi_{0} \sqrt{N}}$. The numbers $m \in\{1: M\}$ and $n \in\{1: N\}$, where $M$ is the total number of sensing operators and $N$ is the dimension of the Hilbert state of the unknown wavefunction. To find the wavefunction $\psi$ we need to (approximately) solve this linear system of equations in the case where $M \ll N$. A nonlinear strategy can be used to recover $\psi$ with a high quality using the idea of compressive sensing (CS). If the wavefunction under the experiment $\psi$ is known to have very few non-zero coefficients under a linear transformation $\mathbf{T}$, it can be reconstructed with a high probability by solving the convex optimization problem [3]

$$
\min _{\psi^{\prime}}\left\|\mathbf{T} \psi^{\prime}\right\|_{\ell_{1}}, \text { subject to } \mathbf{Q} \psi^{\prime}=\phi .
$$

Fig. 1 shows the schematics of the experiment. A vertically polarized Gaussian mode is prepared by spatially filtering a He-Ne laser beam with a single mode fiber and passing it through a polarizer. A random polarization rotation at each point is performed using a spatial light modulator (SLM) in combination with two quarter wave plates (QWP) [4]. To provide a quantitive comparison of the two methods we calculate the fidelity between a retrieved Gaussian state $\left|\psi^{\prime}\right\rangle$ and the state $|\psi\rangle$ from a full pixel-by-pixel scan (See Fig. 1). We prepare a custom target state by illuminating phase mask depicting letters $\mathrm{U}$ and $\mathrm{R}$ with a phase jump of $\pi / 2$ with a Gaussian beam. Figure 2 shows the amplitude and the phase of the reconstructed state with $M / N=20 \%$ of the total measurements. Notice that while the amplitude is relatively uniform, the phase shows the letters $\mathrm{U}$ and $\mathrm{R}$ with a remarkable accuracy.

To conclude, we have demonstrated high fidelity reconstruction of spatial states using the compressive direct measurement (CDM) method. This technique can be used for measurement of high-dimensional quantum states as well as classical applications such as wavefront sensing.

\section{References}

1. J. S. Lundeen, B. Sutherland, A. Patel, C. Stewart, and C. Bamber, "Direct measurement of the quantum wavefunction." Nature 474, 188-191 (2011).

2. Y. Aharonov, D. Albert, and L. Vaidman, "How the result of a measurement of a component of the spin of a spin-1/2 particle can turn out to be 100," Physical Review Letters 60, 1351-1354 (1988).

3. J. Romberg, "Imaging via Compressive Sampling," IEEE Signal Processing Magazine 25, 14-20 (2008).

4. M. Malik, M. Mirhosseini, M. P. J. Lavery, J. Leach, M. J. Padgett, and R. W. Boyd, "Direct measurement of a 27-dimensional orbital-angular-momentum state vector," Nature Communications 5, 3115 (2014). 